\title{
Concomitant Evaluation of Heart Period and QT Interval Variability Spectral Markers to Typify Cardiac Control in Humans and Rats
}

\section{Beatrice De Maria', Vlasta Bari2, Andrea Sgoifo ${ }^{3,4}$, Luca Carnevali3,4, Beatrice Cairo ${ }^{5}$, Emanuele Vaini ${ }^{2}$, Aparecida Maria Cataib, Anielle Cristhine de Medeiros Takahashi6, Laura Adelaide Dalla Vecchia' ${ }^{1}$ and Alberto Porta ${ }^{2,5 *}$}

\begin{abstract}
${ }^{1}$ IRCCS Istituti Clinici Scientifici Maugeri, Milan, Italy, ${ }^{2}$ Department of Cardiothoracic, Vascular Anesthesia and Intensive Care, IRCCS Policlinico San Donato, Milan, Italy, ${ }^{3}$ Stress Physiology Laboratory, Department of Chemistry, Life Sciences and Environmental Sustainability, University of Parma, Parma, Italy, ${ }^{4}$ Microbiome Research Hub, University of Parma, Parma, Italy, ${ }^{5}$ Department of Biomedical Sciences for Health, University of Milan, Milan, Italy, ${ }^{6}$ Department of Physiotherapy, Federal University of São Carlos, São Carlos, Brazil
\end{abstract}

OPEN ACCESS

Edited by:

George E. Billman,

The Ohio State University,

United States

Reviewed by:

Veronique Meijborg

Academic Medical Center (AMC),

Netherlands

Jerzy Sacha,

Opole University of Technology,

Poland

*Correspondence:

Alberto Porta

alberto.porta@unimi.it

Specialty section:

This article was submitted to

Cardiac Electrophysiology,

a section of the journal

Frontiers in Physiology

Received: 09 July 2019 Accepted: 18 November 2019 Published: 29 November 2019

Citation:

De Maria B, Bari V, Sgoifo A

Carnevali L, Cairo B, Vaini E, Catai AM, de Medeiros Takahashi AC, Dalla Vecchia LA and Porta A (2019) Concomitant Evaluation of Heart Period and QT Interval Variability Spectral Markers to Typify Cardiac

Control in Humans and Rats.

Front. Physiol. 10:1478.

doi: 10.3389/fphys.2019.01478
The variability of heart period, measured as the time distance between two consecutive QRS complexes from the electrocardiogram (RR), was exploited to infer cardiac vagal control, while the variability of the duration of the electrical activity of the heart, measured as the time interval from $\mathrm{Q}$-wave onset to T-wave end (QT), was proposed as an indirect index of cardiac sympathetic modulation. This study tests the utility of the concomitant evaluation of RR variability (RRV) and QT variability (QTV) markers in typifying cardiac autonomic control of humans under different experimental conditions and of rat groups featuring documented differences in resting sympatho-vagal balance. We considered: (i) 23 healthy young subjects in resting supine position (REST) undergoing head-up tilt at $45^{\circ}$ (T45) and $90^{\circ}$ (T90) followed by recovery to the supine position; (ii) 9 Wistar (WI) and 14 wild-type Groningen (WT) rats in unstressed conditions, where the WT animals were classified as non-aggressive (non-AGG, $n=9$ ) and aggressive (AGG, $n=5$ ) according to the resident intruder test. In humans, spectral analysis of RRV and QTV was performed over a single stationary sequence of 250 consecutive values. In rats, spectral analysis was iterated over 10-min recordings with a frame length of 250 beats with $80 \%$ overlap and the median of the distribution of the spectral markers was extracted. Over RRV and QTV we computed the power in the low frequency (LF, from 0.04 to $0.15 \mathrm{~Hz}$ in humans and from 0.2 to $0.75 \mathrm{~Hz}$ in rats) band (LFRR and $\left.L F_{Q T}\right)$ and the power in the high frequency (HF, from 0.15 to $0.5 \mathrm{~Hz}$ in humans and from 0.75 to $2.5 \mathrm{~Hz}$ in rats) band $\left(\mathrm{HF}_{\mathrm{RR}}\right.$ and $\left.\mathrm{HF}_{\mathrm{QT}}\right)$. In humans the $\mathrm{HF}_{\mathrm{RR}}$ power was lower during $\mathrm{T} 90$ and higher during recovery compared to REST, while the LF ${ }_{Q T}$ power was higher during T90. In rats the $\mathrm{HF}_{\mathrm{RR}}$ power was lower in $\mathrm{WT}$ rats compared to $\mathrm{WI}$ rats and the LF $F_{\mathrm{QT}}$ power was higher in AGG than in non-AGG animals. We concluded that RRV and QTV provide complementary information in describing the functioning of vagal and sympathetic limbs of the autonomic nervous system in humans and rats.

Keywords: power spectral analysis, heart rate variability, QTV, ventricular repolarization, autonomic nervous system, wild-type rat, Wistar, head-up tilt 


\section{INTRODUCTION}

Heart period, measured as the time distance between two consecutive QRS complexes from the electrocardiogram (RR), exhibits spontaneous fluctuations usually referred to as $R R$ variability (RRV). The analysis of RRV provides some markers that have been found useful to infer the state of the cardiac autonomic control (Task Force of the European Society of Cardiology and the North American Society of Pacing and Electrophysiology,, 1996). Short-term RRV markers in humans are mainly associated with vagal modulation given that the magnitude of RR changes is dramatically reduced by full vagal blockade (Pomeranz et al., 1985). This consideration holds not only in humans but also in rats (Japundzic et al., 1990; Cerutti et al., 1991; Silva et al., 2017) and this analogy strengthened the use of rats as an animal model of human autonomic cardiac control. The amplitude of the respiratory sinus arrhythmia is one of the most utilized RRV indexes to typify cardiac vagal control (Hirsch and Bishop, 1981): it is frequently estimated via spectral analysis as the power of RRV in the high frequency (HF) band in both humans and rats, even though the definition of the HF band was adapted to account for the differences between the respiratory rates in the two species, namely from 0.15 to $0.5 \mathrm{~Hz}$ in humans and from 0.75 to $2.5 \mathrm{~Hz}$ in rats (Japundzic et al., 1990; Cerutti et al., 1991; Rubini et al., 1993). In particular, in humans the HF power of RRV is known to decrease during physiological conditions characterized by sympathetic activation and vagal withdrawal, such as during graded orthostatic challenge (Montano et al., 1994; Cooke et al., 1999; Porta et al., 2011; Marchi et al., 2016) or physical exercise (Shin et al., 1995a,b; Brenner et al., 1997; Porta et al., 2018). Similarly, in rats the HF power of RRV was utilized to typify the autonomic response to several types of stressors either pharmacological, interventional, or social (Akselrod et al., 1987; Japundzic et al., 1990; Cerutti et al., 1991; Rubini et al., 1993; Stauss et al., 1997; Sgoifo et al., 1998, 1999; Jaenisch et al., 2011; Carnevali et al., 2013; Carnevali and Sgoifo, 2014; Silva et al., 2016, 2017).

More recently, in parallel with the more traditional RRV analysis, the variability of the overall duration of the electrical activity of the heart, comprising depolarization and repolarization periods, usually quantified as the time interval from Q-wave onset to $\mathrm{T}$-wave end (QT) from the electrocardiogram, has been proposed and validated as a marker of cardiac sympathetic control in humans (Berger, 2009; Malik, 2009; Porta et al., 2010; Baumert et al., 2016). QT variability (QTV) markers computed in the low frequency (LF) band (i.e., from 0.04 to $0.15 \mathrm{~Hz}$ in humans) have been found to increase in situations where sympatho-vagal balance is shifted toward sympathetic activation and vagal withdrawal, especially when the sympathetic drive is particularly high (Lombardi et al., 1996; Porta et al., 1998a, 2010, 2011; Yeragani et al., 2000a,b; Piccirillo et al., 2001, 2006; Bar et al., 2007; Baumert et al., 2008, 2011; El-Hamad et al., 2015), with relevant clinical consequences in risk stratification (Berger et al., 1997; Atiga et al., 1998; Porta et al., 2015). Conversely, no information was provided about the possibility to use QTV in rats, mainly because of the technical difficulties in reliably assessing QT fluctuations due to the very limited signal-to-noise ratio of QTV (Laguna et al., 1990; Speranza et al., 1993; Lombardi et al., 1996; Porta et al., $1998 \mathrm{~b})$ and the peculiarities of cardiac repolarization in rodents (Conrath and Opthof, 2006; Fabritz et al., 2010; Speerschneider and Thomsen, 2013; Boukens et al., 2014).

The aim of the present study is to propose the concomitant evaluation of RRV and QTV to provide a more complete view on cardiac autonomic control and to test whether this strategy could be fruitfully exploited in both humans and rats. The hypothesis of the study is that the concomitant evaluation of RRV and QTV markers can describe simultaneously cardiac vagal control via the analysis of the RRV and cardiac sympathetic regulation via the analysis of the QTV in both humans and rats. In humans we evaluated two situations of sympathetic activation and vagal withdrawal of different intensities, namely head-up tilt at $45^{\circ}$ and $90^{\circ}$ (Montano et al., 1994), and the following recovery to supine position during which a progressive decline of sympathetic control and a gradual vagal rebound are expected. In rats, we considered two strains with documented differences in resting cardiac sympatho-vagal balance, namely the Wistar (WI) and wild-type Groningen (WT) rats (Carnevali and Sgoifo, 2014), and, within the WT population, two subgroups featuring opposite levels of aggressiveness that have been linked to different states of the cardiac autonomic control (Carnevali et al., 2013).

\section{MATERIALS AND METHODS}

\section{Experimental Protocol on Humans}

We studied 23 young healthy volunteers $(11$ males, age: $26.3 \pm 5.6$ years). A detailed medical history and examination excluded the evidence of any disease. The subjects did not take any medication and consume any caffeine or alcoholcontaining beverages in the $24 \mathrm{~h}$ before the recording session. Each subject underwent two consecutive head-up tilt tests with different table inclination angles, namely $45^{\circ}$ (T45) and $90^{\circ}$ (T90). T45 and T90 sessions were carried out in a random order, lasted $10 \mathrm{~min}$ and were followed by $40 \mathrm{~min}$ of recovery (R45 and R90, respectively) starting when the tilt table was moved back to the horizontal position. The first tilt session was preceded by a 10 -min recording period in supine position (REST). Subjects lay on the tilt table supported by two belts at the level of the thigh and waist, respectively, and with both feet touching the footrest of the tilt table. During the recording sessions, subjects breathed spontaneously but were not allowed to talk. The electrocardiographic activity from a modified lead II was recorded (Biosignal Conditioning Device, Marazza, Monza, Italy) throughout all the experimental sessions and sampled at $1000 \mathrm{~Hz}$. Attention was paid during the positioning of the electrodes to prevent flat or biphasic T-waves. All subjects were able to complete the protocol without experiencing any sign of presyncope. The duration of the phases was never varied.

Informed consent was obtained from all subjects before taking part in the study. The study adheres to the principles of the Declaration of Helsinki for medical research involving human subjects. The Human Research and Ethical Review Board of the L. Sacco Hospital, Milan, Italy, approved the protocol. 


\section{Experimental Protocol on Rats}

We studied two different strains of rats: 9 male WI rats (age: $5.5 \pm 0.5$ months; weight: $436 \pm 34 \mathrm{~g}$ ) and 14 male WT rats (age: $4.4 \pm 0.5$ months; weight: $395 \pm 40 \mathrm{~g}$ ). Initially WT rats were classified into non-aggressive (non-AGG, $n=5$ ) and aggressive (AGG, $n=9$ ) WT rats, according to the resident intruder test described in Carnevali et al. (2013).

After the preliminary behavioral tests in WT rats, all rats were implanted, under tiletamine hydrochloride plus zolazepam hydrochloride anesthesia (Zoletil Virbac, France, $20 \mathrm{mg} \mathrm{kg}^{-1}$ ), with radiotelemetric transmitters (TA11CTA-F40, Data Sciences International, St. Paul, MN, United States) for the recording of the cardiac electrical activity. Electrocardiograms were picked up by platform receivers (RPC-1, Data Sciences Int., St. Paul, MN, United States) located under the animal's cage and acquired via ART-Gold 4.2 data acquisition system (Data Sciences International, St. Paul, MN, United States) at a sampling rate of $1000 \mathrm{~Hz}$. Animals were individually housed and kept in rooms with controlled temperature $\left(22 \pm 2^{\circ} \mathrm{C}\right)$ and lighting (lights on from 7:00 P.M. to 7:00 A.M.). After a 14-day recovery period from surgery, electrocardiograms were recorded in all rats for $1 \mathrm{~h}$ during the dark (active) phase of the light-dark cycle (i.e., between 10:00 A.M. and 11:00 A.M.) on different days.

The experimental protocol was approved by the Veterinarian Animal Care and Use Committee of the University of Parma, Parma, Italy, and the animals were cared in accordance with the European Community Council Directives of 22 September 2010 (2010/63/UE).

\section{Extraction of the Beat-to-Beat RRV and QTV Series}

The electrocardiographic traces recorded in both healthy humans and rats were processed with a software, developed in house, automatically measuring RR and QT (Porta et al., 1998b). The peak of the QRS complex (i.e., the R-wave) was automatically located via a method based on a threshold on the first derivative of the electrocardiogram. The peak of the QRS complex was fixed via parabolic interpolation. The RR was measured as the time distance between two consecutive QRS complex peaks. The QT was approximated as the time interval between the peak of the QRS complex and the T-wave offset (RTend) (Porta et al., 2010, 2011). The end of the T-wave was automatically delineated where the absolute value of the first derivative calculated on T-wave downslope became smaller than $30 \%$ of the absolute value of the steepest slope of the T-wave. Figure 1 shows an example of the automatic detection of the T-wave end in a healthy subject (top panel), a WI rat (middle panel), and a WT rat (bottom panel). The detections of the QRS complex were visually checked and corrected in case of identification errors and in this case the T-wave offset delineation procedure was run again starting from the new position of the QRS complex. T-wave end detections were checked to assure the quality of the T-wave delineation. Problematic T-wave morphologies such as biphasic shapes were not observed and the first return to the isoelectric line after the onset of the T-wave always denotes the offset of the repolarization period in both humans and rats. The effects

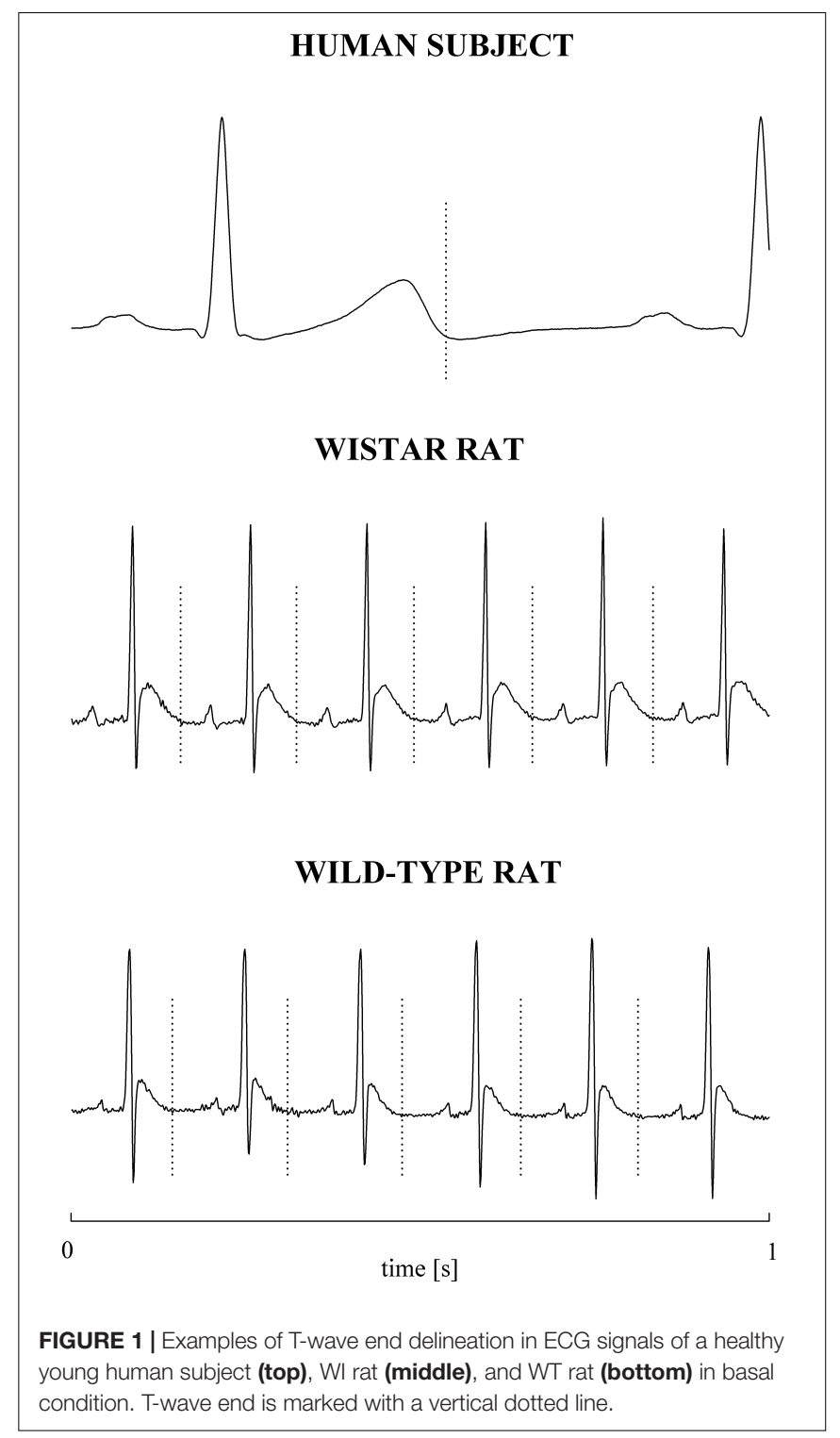

of isolated ectopic beats on RR and QT beat-to-beat series were corrected by means of cubic spline interpolation starting from the RR and QT measures unaffected by non-sinus cardiac beats. Corrections never exceeded the $5 \%$ of the total beats. Within each experimental session of the human protocol (i.e., REST, T45, R45, T90, and R90) segments of 250 consecutive RR and QT measures were selected. Stationarity of the selected sequences was tested according to Magagnin et al. (2011). The first stationary sequence found $3 \mathrm{~min}$ after the onset of posture changes was taken as the representative segment during T45 and T90 sessions and the first stationary sequence $10 \mathrm{~min}$ after returning to the supine position after head-up tilt was taken as the representative segment during R45 and R90 sessions. As to the animal protocol, a 10-min segment was selected in a random position within the overall recording session. The analysis was carried out over the 10-min segments divided into adjacent windows of 250 consecutive RR and QT measures with $80 \%$ overlap. The median 
of the distribution was chosen as the representative value of the whole series. Figure 2 shows some examples of beat-to-beat RRV and QTV series derived from a human subject at REST (Figures 2A,D), from a WI rat (Figures 2B,E) and a WT rat (Figures 2C,F) in unstressed conditions.

\section{Time and Frequency Domain RRV and QTV Analyses}

In the time domain, we computed the mean of RR and QT beat-to-beat series ( $\mu_{\mathrm{RR}}$ and $\mu_{\mathrm{QT}}$, respectively). $\mu_{\mathrm{RR}}$ and $\mu_{\mathrm{QT}}$ were expressed in ms. Linear detrending procedure, subtracting from the original series the best fit linear trend, was exploited to prevent the drift of the mean and favor stationarity. After linear detrending of the series, the variances of RR and QT beat-to-beat series $\left(\sigma_{\mathrm{RR}}^{2}\right.$ and $\sigma_{\mathrm{QT}}^{2}$, respectively) were calculated and expressed in $\mathrm{ms}^{2}$. Parametric power spectral analysis was performed. RRV and QTV series were modeled as realizations of an autoregressive process. The coefficients of the autoregressive process and the variance of the white noise corrupting the determinist part of the process were estimated via least squares method solved via the Levinson-Durbin recursion (Kay and Marple, 1981). The number of coefficients was optimized via Akaike's (1974) criterion within the range from 10 to 16 . Power spectral density was decomposed into power spectral components (Baselli et al., 1997), classified as LF or HF component, according to their central frequency. The LF band range was $0.04-0.15 \mathrm{~Hz}$ for humans (Task Force of the European Society of Cardiology and the North American Society of Pacing and Electrophysiology, 1996) and 0.2-0.75 Hz for rats (Carnevali et al., 2013), while the $\mathrm{HF}$ band range was $0.15-0.5 \mathrm{~Hz}$ for humans (Task Force of the European Society of Cardiology and the North American Society of Pacing and Electrophysiology,, 1996) and $0.75-2.5 \mathrm{~Hz}$ for rats (Carnevali et al., 2013). The sum of the absolute power of all HF components of the $\mathrm{RR}$ series was termed as $\mathrm{HF}_{\mathrm{RR}}$ and considered to be an index of vagal modulation directed to the sinus node (Pomeranz et al., 1985), whereas the sum of the absolute power of all LF components of the QT series was labeled LF $\mathrm{QT}_{\mathrm{T}}$ and considered to be an index of sympathetic modulation directed to the heart (Porta et al., 2010, 2011; Baumert et al., 2011; El-Hamad et al., 2015). The power of RRV in the LF band, indicated as $L_{R R}$, and the power of the QTV in the $\mathrm{HF}$ band, labeled as $\mathrm{HF}_{\mathrm{QT}}$, were computed as well. $\mathrm{LF}_{\mathrm{RR}}, \mathrm{HF}_{\mathrm{RR}}, \mathrm{LF}_{\mathrm{QT}}$, and $\mathrm{HF}_{\mathrm{QT}}$ indexes were given in absolute units and expressed in $\mathrm{ms}^{2}$. Spectral analysis was carried out over the linearly detrended RRV and QTV series.

\section{Statistical Analysis}

In the human protocol one-way repeated measures analysis of variance (Dunnett's test for multiple comparisons) was performed to check the significance of the differences of T45, R45, T90, and R90 versus REST. If the Kolmogorov-Smirnov normality test was not passed, Friedman one-way repeated measures analysis of variance on ranks (Dunnett's test for multiple comparisons) was carried out. In the animal protocol unpaired $t$-test was performed to assess the significance of the differences between the strains (WI versus WT) and subgroups (AGG versus non-AGG). If the Kolmogorov-Smirnov normality test was not passed, Mann-Whitney rank sum test was carried out. Statistical analysis was carried out using a commercial statistical program (Sigmaplot, Systat Software, Inc., Chicago, IL, United States, version 11.0). A $p<0.05$ was always considered as significant.

\section{RESULTS}

Box-and-whisker plots of Figure 3 show the results of RRV (Figures 3A-C) and QTV (Figures 3D-F) analyses performed on human data as a function of the experimental condition (i.e., REST, T45, R45, T90, and R90). Compared to REST, $\mu_{\mathrm{RR}}$ decreased during both T45 and T90, while it was unchanged during R45 and R90 (Figure 3A). $\sigma_{\mathrm{RR}}^{2}$ was significantly higher during both R45 and R90 and was not affected by the orthostatic challenge (Figure 3B). $\mathrm{HF}_{\mathrm{RR}}$ power significantly decreased during T90 and increased during R90 compared to REST (Figure 3C). $\mu_{\mathrm{QT}}$ was significantly reduced during both $\mathrm{T} 45$ and T90 and did not vary during R45 and R90 (Figure 3D). $\sigma_{\mathrm{QT}}^{2}$ did not change with the experimental condition (Figure $3 \mathrm{E}$ ). $\mathrm{LF}_{\mathrm{QT}}$ power increased during T90 and remained stable in all the other experimental conditions (Figure 3F). Results relevant to time and frequency domain RRV and QTV markers derived from the experimental protocol on humans are summarized in Table $\mathbf{1}$. The same table reports the $\mathrm{LF}_{\mathrm{RR}}$ and $\mathrm{HF}_{\mathrm{QT}}$ powers as well. Both these latter markers did not vary with the experimental condition.

Box-and-whisker plots of Figure 4 show the results of RRV (Figures 4A-C) and QTV (Figures 4D-F) analyses performed on data derived from WI and WT rats. $\mu_{\mathrm{RR}}$ (Figure 4A) and $\sigma_{\mathrm{RR}}^{2}$ (Figure 4B) were similar between the two strains, while the $\mathrm{HF}_{\mathrm{RR}}$ power (Figure 4C) was higher in WI compared to WT rats. $\mu_{\mathrm{QT}}$ (Figure 4D) was longer in WI rats, while no strain differences in $\sigma_{\mathrm{QT}}^{2}$ (Figure $4 \mathrm{E}$ ) and $\mathrm{LF}_{\mathrm{QT}}$ power (Figure $4 \mathrm{~F}$ ) were observed. Figure 5 has the same structure as Figure 4 but it shows the results of RRV (Figures 5A-C) and QTV (Figures 5DF) analyses performed on data obtained from WT rats that were classified as non-AGG and AGG. $\mu_{\mathrm{RR}}$ (Figure 5A), $\sigma_{\mathrm{RR}}^{2}$ (Figure 5B), $\mathrm{HF}_{\mathrm{RR}}$ (Figure 5C), and $\mu_{\mathrm{QT}}$ (Figure 5D) did not differentiate the two subgroups. On the contrary, $\sigma_{\mathrm{QT}}^{2}$ (Figure 5E) and $\mathrm{LF}_{\mathrm{QT}}$ (Figure 5F) were able to separate the two groups of WT rats, being both $\sigma_{\mathrm{QT}}^{2}$ and $\mathrm{LF}_{\mathrm{QT}}$ power higher in AGG than in non-AGG animals. RRV and QTV markers derived from the experimental protocol on rats are summarized in Tables 2, 3. These tables reported $\mathrm{LF}_{\mathrm{RR}}$ and $\mathrm{HF}_{\mathrm{QT}}$ powers as well. Both these markers were similar in WI and WT animals (Table 2) and they were not able to distinguish non-AGG from AGG animals (Table 3).

\section{DISCUSSION}

To the best of our knowledge this is the first study in which QTV parameters were evaluated in rats concomitantly with traditional RRV measures for the assessment of cardiac autonomic control and a parallel between human and rat RRV and QTV markers was drawn. The most important findings of this study can be 

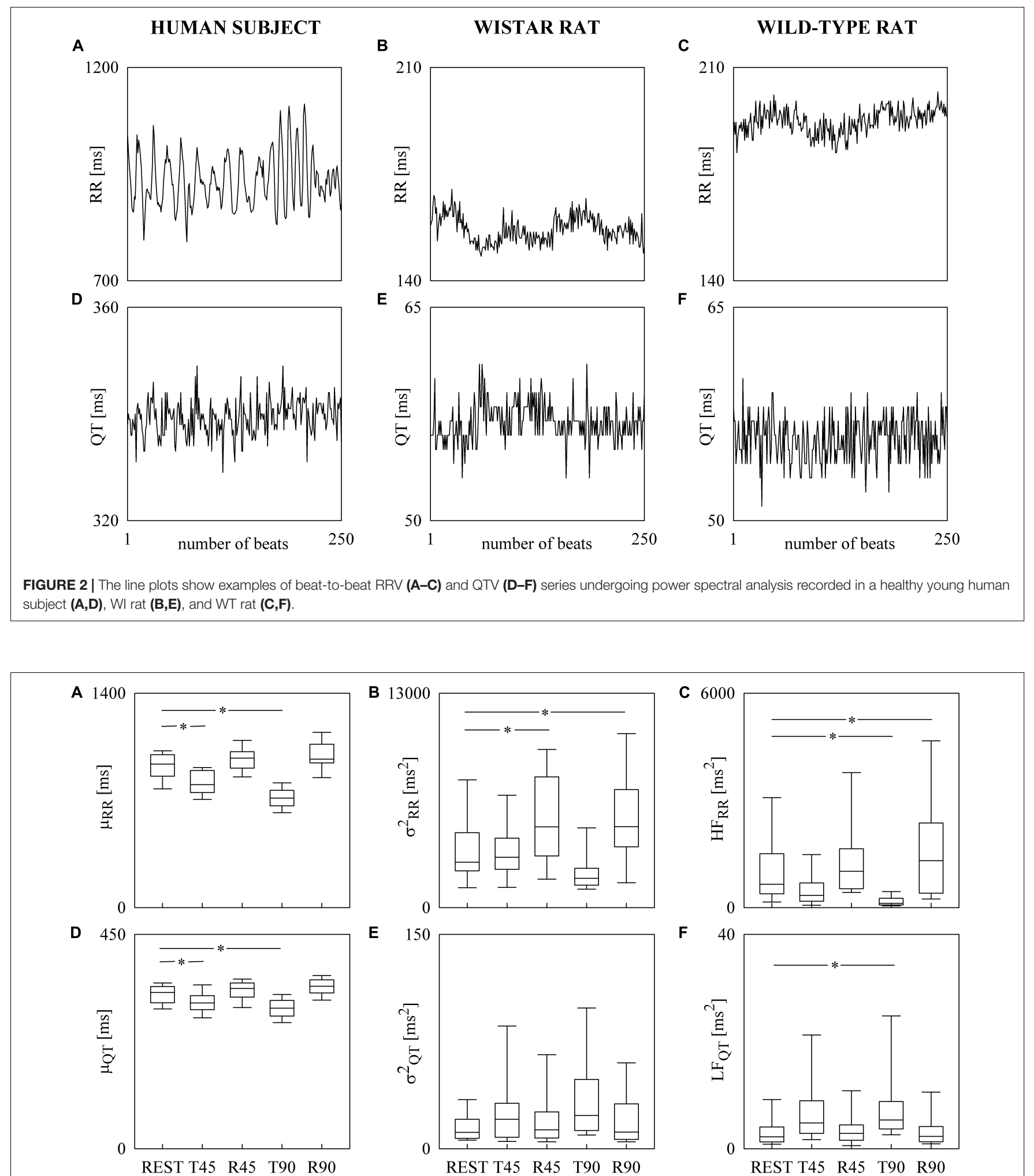

FIGURE 3 | The box-and-whisker graphs show the results of the time and frequency domain analyses of RRV and QTV in healthy young humans. The $\mu_{\mathrm{RR}}$ (A), $\sigma_{\mathrm{RR}}^{2}$ (B), $\mathrm{HF}_{\mathrm{RR}}(\mathbf{C}), \mu_{Q T}$ (D), $\sigma_{\mathrm{QT}}^{2}(\mathbf{E})$, and $L F_{\mathrm{QT}}(\mathbf{F})$ are given as a function of the experimental condition (i.e., REST, T45, R45, T90, and R90). Box height represents the interquartile range, median is marked with a horizontal solid segment, and whiskers denote the 10 th and 90 th percentile. The symbol $*$ indicates a $p<0.05$ versus REST. 
TABLE 1 | Results of the time and frequency domain analyses of RRV and QTV in healthy young subjects.

\begin{tabular}{|c|c|c|c|c|c|}
\hline Index & REST & T45 & R45 & T90 & R90 \\
\hline$\mu_{\mathrm{RR}}(\mathrm{ms})$ & $937.18(135.11)$ & $802.56(142.08)^{*}$ & $975.94(97.25)$ & $715.18(96.47)^{*}$ & 969.17 (117.36) \\
\hline$\sigma_{\mathrm{RR}}^{2}\left(m s^{2}\right)$ & $2755.2(2083.51)$ & 3049.86 (1596.87) & $4894.99(4585.21)^{*}$ & $1773.36(940.24)$ & $4904.22(3447.03)^{*}$ \\
\hline $\mathrm{LF}_{\mathrm{RR}}\left(\mathrm{ms}^{2}\right)$ & 952.56 (1219.09) & 1180.74 (1161.83) & 1694.45 (1840.67) & 890.56 (766.05) & 1372.49 (1741.51) \\
\hline$H F_{R R}\left(m s^{2}\right)$ & 651.6 (1095.69) & 342.08 (479.25) & 1016.27 (1040.69) & $117.27(182.51)^{*}$ & $1312.98(1651.28)^{*}$ \\
\hline$\mu_{\mathrm{QT}}(\mathrm{ms})$ & 327.91 (33.6) & $306.05(26.65)^{*}$ & $336.25(27.46)$ & $295.09(30.19)^{*}$ & 341.03 (26.73) \\
\hline$\sigma_{\mathrm{QT}}^{2}\left(\mathrm{~ms}^{2}\right)$ & 11.43 (12.29) & 20.67 (23.11) & 13.13 (16.72) & 23.21 (33.49) & 11.59 (23.18) \\
\hline $\mathrm{LF}_{\mathrm{QT}}\left(\mathrm{ms}^{2}\right)$ & 2.2 (3.98) & $4.8(5.71)$ & $2.86(2.76)$ & $5.36(4.78)^{*}$ & $2.29(2.5)$ \\
\hline $\mathrm{HF}_{\mathrm{QT}}\left(\mathrm{ms}^{2}\right)$ & $4.45(5.91)$ & 7.43 (12.09) & $3.34(9.89)$ & 8.62 (11.9) & $4.09(11.28)$ \\
\hline
\end{tabular}

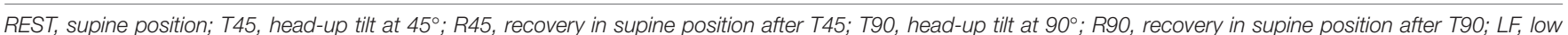

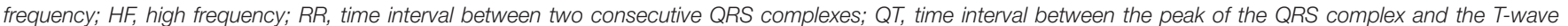

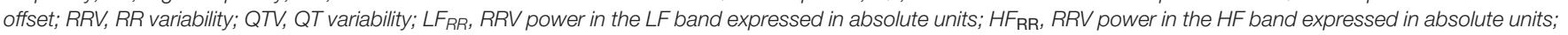

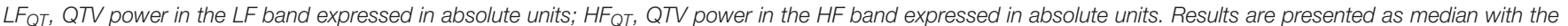
interquartile range in round brackets. The symbol * indicates a $p<0.05$ versus REST.

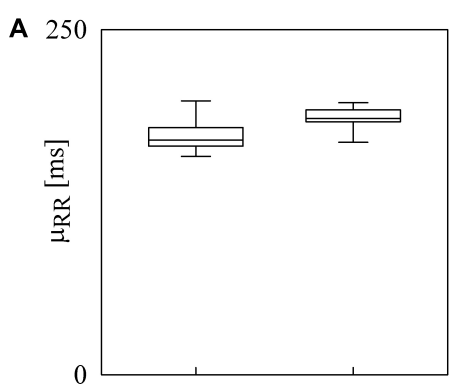

D

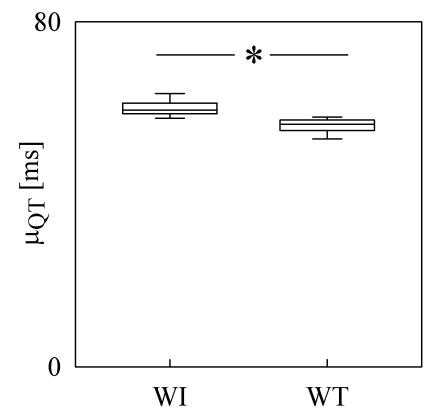

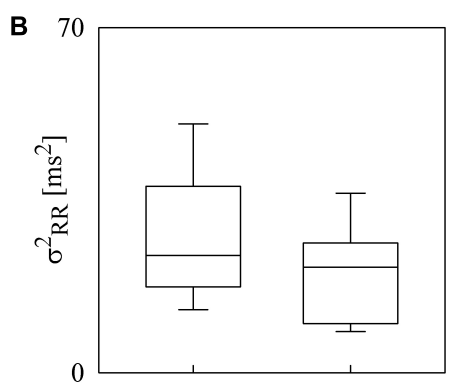

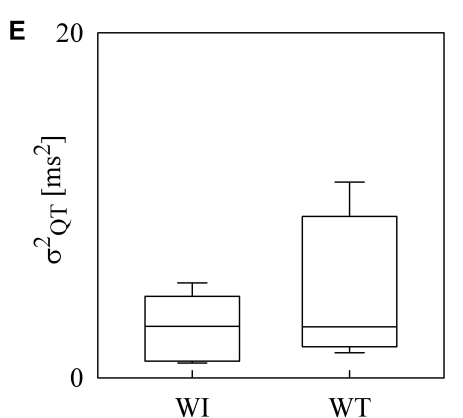

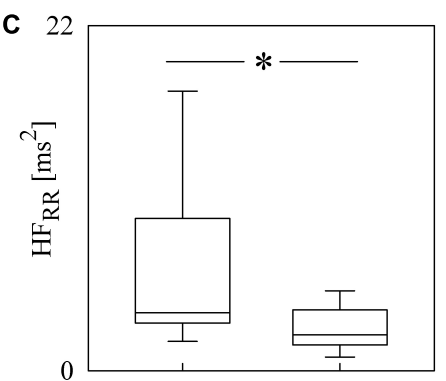

$\mathbf{F}$

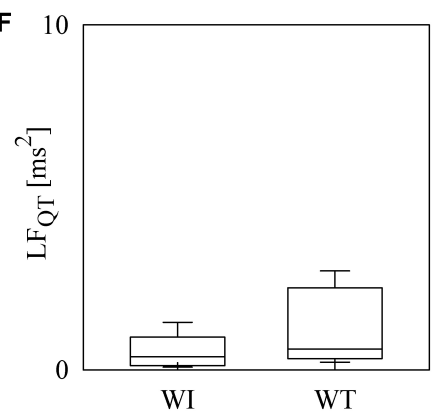

FIGURE 4 | The box-and-whisker graphs show the results of the time and frequency domain analyses of RRV and QTV in rats. The $\mu_{R R}(\mathbf{A}), \sigma_{R R}^{2}(\mathbf{B}), H_{R R}(\mathbf{C}), \mu_{Q T}$ (D), $\sigma_{Q T}^{2}(\mathbf{E})$, and $L F_{Q T}(\mathbf{F})$ are given as a function of the strain (i.e., Wl and WT). Box height represents the interquartile range, median is marked with a horizontal solid segment, and whiskers denote the 10th and 90th percentile. The symbol * indicates a $p<0.05$ versus WI rats.

summarized as follows: (i) RRV is descriptive of the cardiac vagal regulation in both humans and rats; (ii) QTV is representative of cardiac sympathetic control in both humans and rats; (iii) results of RRV and QTV should be simultaneously considered to more deeply describe cardiac autonomic control in both humans and rats.

\section{RRV and QTV Provide Complementary Information About Cardiac Autonomic Control in Humans and Rats}

One of the major difficulties in exploiting RRV and spectral markers derived from RRV analysis to comprehensively characterize cardiac autonomic control is the strong link of RRV with the variation of vagal autonomic outflow, while its sensitivity to changes of the activity of the sympathetic autonomic limb is more limited. Indeed, since the initial studies on RRV (Akselrod et al., 1981; Pomeranz et al., 1985) it is well-known that the $\mathrm{HF}_{\mathrm{RR}}$ power is completely abolished by full vagal blockade carried out via a high dose of atropine and that the same pharmacological challenge affects remarkably the $L_{R R}$ power as well. This observation suggested that the $H_{R R}$ power is a genuine marker of vagal modulation directed to the sinus node, while the $\mathrm{LF}_{\mathrm{RR}}$ power results from the changes of the activity of both sympathetic and vagal limbs of the autonomic nervous system (Akselrod et al., 1981; Pomeranz et al., 1985). Normalization strategies attempted to limit the dependence of the $L_{\mathrm{RR}}$ power on cardiac vagal control. For example, the ratio of the $L_{\mathrm{RR}}$ power to $\sigma_{\mathrm{RR}}^{2}$ minus the RRV power in the very LF band, known as $L_{R R}$ power expressed 


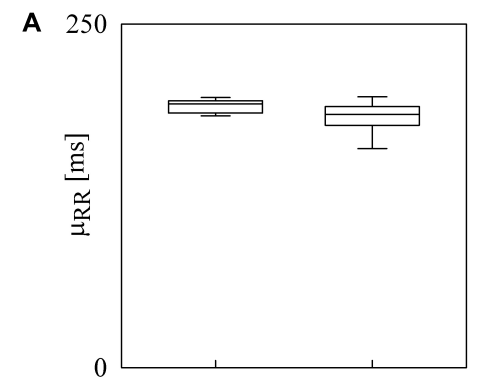

D

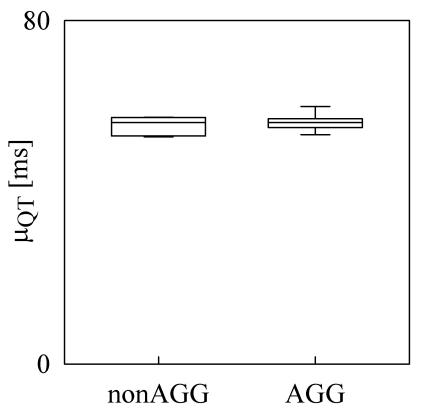

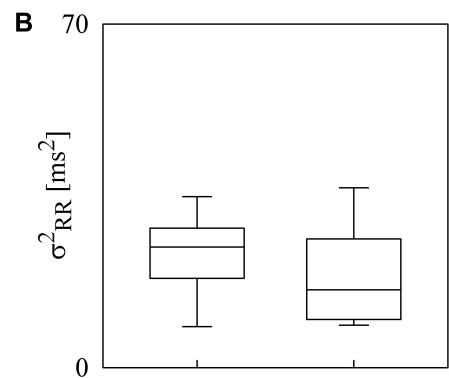

E

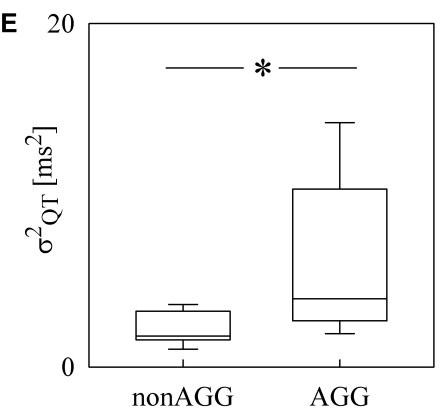

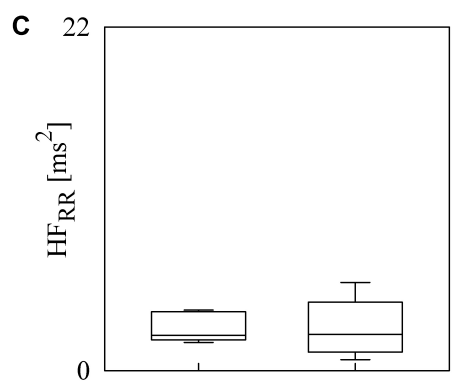

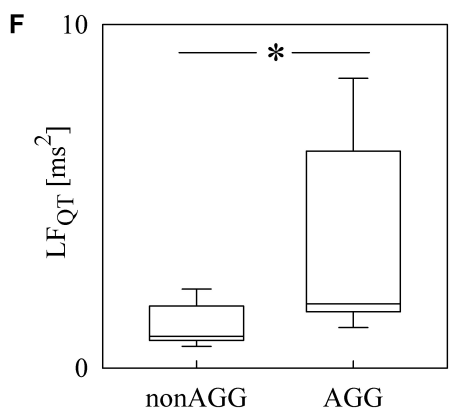

FIGURE 5 | The box-and-whisker graphs show the results of the time and frequency domain analyses of RRV and QTV in WT rats classified into non-AGG and AGG animals. The $\mu_{R R}$ (A), $\sigma_{R R}^{2}$ (B), $H F_{R R}$ (C), $\mu_{Q T}$ (D), $\sigma_{Q T}^{2}$ (E), and $L F_{Q T}(\mathbf{F})$ are given as a function of the WT subcategory (i.e., non-AGG and AGG). Box height represents the interquartile range, median is marked with a horizontal solid segment, and whiskers denote the 10th and 90th percentile. The symbol * indicates a $p<0.05$ versus non-AGG animals.

TABLE 2 | Results of time and frequency domain analyses of RRV and QTV in WI and WT rats.

\begin{tabular}{lcc}
\hline Index & WI & WT \\
\hline$\mu_{\mathrm{RR}}(\mathrm{ms})$ & $169.99(7.37)$ & $185.6(8.31)$ \\
$\sigma_{\mathrm{RR}}^{2}\left(\mathrm{~ms}^{2}\right)$ & $23.76(19.62)$ & $21.38(15.5)$ \\
$\mathrm{LF}_{\mathrm{RR}}\left(\mathrm{ms}^{2}\right)$ & $1.22(1.4)$ & $0.58(0.37)$ \\
$\mathrm{H} F_{\mathrm{RR}}\left(\mathrm{ms}^{2}\right)$ & $3.69(4.63)$ & $2.29(2.16)^{*}$ \\
$\mu_{\mathrm{QT}}\left(\mathrm{ms}^{2}\right.$ & $59.54(2.21)$ & $56.23(2.32)^{*}$ \\
$\sigma_{\mathrm{QT}}^{2}\left(\mathrm{~ms}^{2}\right)$ & $2.98(3.49)$ & $2.95(6.01)$ \\
$\mathrm{LF}_{\mathrm{QT}}\left(\mathrm{ms}^{2}\right)$ & $0.38(0.78)$ & $0.6(1.63)$ \\
$\mathrm{H} F_{\mathrm{QT}}\left(\mathrm{ms}^{2}\right)$ & $1.36(1.93)$ & $1.68(3.76)$
\end{tabular}

WI, Wistar rats; WT, wild-type Groningen rats; LF, low frequency; $H F$, high frequency; RR, time interval between two consecutive QRS complexes; $Q T$, time interval between the peak of the QRS complex and the T-wave offset; RRV, RR variability; $Q T V, Q T$ variability; $L F_{R R}, R R V$ power in the $L F$ band expressed in absolute units; $H F_{R R}$, RRV power in the HF band expressed in absolute units; $L F_{Q T}$, QTV power in the LF band expressed in absolute units; $H F_{Q T}$, QTV power in the HF band expressed in absolute units. Results are presented as median with the interquartile range in round brackets. The symbol * indicates a $p<0.05$ versus WI.

in normalized units (Pagani et al., 1986), is one of the most frequently exploited normalized RRV indexes. The attempts of normalizing frequency domain markers of RRV to achieve a more genuine marker of sympathetic control generated some controversies (Eckberg, 1997; Pagani et al., 1997; Billman, 2013; Reyes del Paso et al., 2013). Among the most controversial issues there is the non-zero value of normalized $L_{\mathrm{RR}}$ power after full vagal blockade in presence of null $R R$ changes and the strict link between normalized $\mathrm{LF}_{\mathrm{RR}}$ and normalized $\mathrm{HF}_{\mathrm{RR}}$ powers given that their sum is 100 (Eckberg, 1997). The final result is
TABLE 3 | Results of time and frequency domain analyses of RRV and QTV in WT rats classified as non-AGG and $A G G$ animals.

\begin{tabular}{lcc}
\hline Index & non-AGG & AGG \\
\hline$\mu_{\mathrm{RR}}(\mathrm{ms})$ & $191.9(7.27)$ & $184.22(11.4)$ \\
$\sigma_{\mathrm{RR}}^{2}\left(\mathrm{~ms}^{2}\right)$ & $24.57(4.83)$ & $15.86(12.84)$ \\
$\mathrm{LF}_{\mathrm{RR}}\left(\mathrm{ms}^{2}\right)$ & $0.66(0.24)$ & $0.53(0.45)$ \\
$\mathrm{HF}_{\mathrm{RR}}\left(\mathrm{ms}^{2}\right)$ & $2.26(1.71)$ & $2.32(2.89)$ \\
$\mu_{\mathrm{QT}}(\mathrm{ms})$ & $56.21(4.2)$ & $56.25(1.9)$ \\
$\sigma_{\mathrm{QT}}^{2}\left(\mathrm{~ms}^{2}\right)$ & $1.8(1.37)$ & $3.98(7.5)^{*}$ \\
$\mathrm{LF}$ & $0.3(0.54)$ & $0.67(1.96)^{*}$ \\
$\mathrm{HF}_{\mathrm{QT}}\left(\mathrm{ms}^{2}\right)$ & $0.92(0.78)$ & $1.87(4.59)$
\end{tabular}

WT, wild-type Groningen rats; non-AGG, non-aggressive WT rats; AGG, aggressive WT rats; $L F$, low frequency; $H F$, high frequency; $R R$, time interval between two consecutive QRS complexes; QT, time interval between the peak of the QRS complex and the T-wave offset; $R R V, R R$ variability; $Q T V, Q T$ variability; $L F_{R R}, R R V$ power in the LF band expressed in absolute units; $H F_{R R}$, RRV power in the HF band expressed in absolute units; $L F_{Q T}$, QTV power in the LF band expressed in absolute units; $H F_{Q T}, Q T V$ power in the HF band expressed in absolute units. Results are presented as median with the interquartile range in round brackets. The symbol * indicates a $p<0.05$ versus non-AGG.

that no normalization procedure solved the original problem due to the inherent contribution of vagal limb to RRV in the LF band (Akselrod et al., 1981; Pomeranz et al., 1985). More recently, some studies on QTV have suggested the possibility of monitoring cardiac sympathetic control via markers extracted from QTV (Porta et al., 1998a, 2010; Berger, 2009; Malik, 2009; El-Hamad et al., 2015; Baumert et al., 2016) and have outlined the clinical relevance of this approach in pathological populations and risk stratification (Berger et al., 1997; Atiga et al., 1998; Baumert et al., 2008, 2011; Bari et al., 2014; Porta et al., 2015). 
A pragmatic route to face the issue generated by the debate on the use of RRV markers in the frequency domain was made operational in Porta et al. (2015) who proposed the simultaneous exploitation of RRV and QTV to derive a frequency domain description of the cardiac vagal control via the $H_{\mathrm{RR}}$ power and of the cardiac sympathetic control via the LF $\mathrm{QT}_{\mathrm{T}}$ power. The strategy proposed in Porta et al. (2015) was tested in this study in humans during an experimental protocol evoking sympathetic activation and vagal withdrawal (i.e., head-up tilt) and the progressive sympathetic regulation departure and vagal control rebound during recovery after the postural challenge (Montano et al., 1994; Cooke et al., 1999; Porta et al., 2011; Marchi et al., 2016) and in rats featuring documented differences in cardiac sympatho-vagal balance at baseline (Carnevali et al., 2013; Carnevali and Sgoifo, 2014). The present study outlines the ability of the simultaneous exploitation of the $\mathrm{HF}_{\mathrm{RR}}$ and $\mathrm{LF}_{\mathrm{QT}}$ markers in typifying state- and trait-related modifications of the cardiac autonomic regulation in human and animal experiments. In the human protocol the significant decrease of the $H_{R R}$ marker during T90 and the concomitant increase of $\mathrm{LF}_{\mathrm{QT}}$ power suggest, respectively, a reduced vagal and an augmented sympathetic controls as it is expected in response to the postural challenge (Montano et al., 1994; Cooke et al., 1999; Porta et al., 2011; Marchi et al., 2016). The specific ability of the $H_{R R}$ marker in tracking the cardiac vagal control was emphasized by the particular design of the experimental protocol in humans considering the period of recovery after the postural challenge. Indeed, the greater cardiac vagal regulation regaining after T90 was stressed by the increase of the $\mathrm{HF}_{\mathrm{RR}}$ power above the levels observed at REST. The independence of the $\mathrm{LF}_{\mathrm{QT}}$ power from the level of cardiac vagal control was supported by the stable values of this index during recovery compared to REST, thus stressing the complementary information that can be derived from the joint use of $\mathrm{HF}_{\mathrm{RR}}$ and $\mathrm{LF}_{\mathrm{QT}}$ markers. The strategy proposing the concomitant use of $\mathrm{HF}_{\mathrm{RR}}$ and $\mathrm{LF}_{\mathrm{QT}}$ powers excludes the utilization of the $L_{\mathrm{RR}}$ power due to its mixed nature and that of the $\mathrm{HF}_{\mathrm{QT}}$ power due to its non-autonomic origin. The mixed origin of the $L_{\mathrm{RR}}$ power is supported by the present study as well: indeed, the constancy of the $L_{\mathrm{RR}}$ power as a function of the experimental condition in the head-up tilt protocol and the inability of the $L_{R R}$ power to distinguish non-AGG from AGG rats is in agreement with a simultaneous increase of sympathetic modulation and a decrease of the vagal one (Porta et al., 2011). The non-autonomic origin of the $\mathrm{HF}_{\mathrm{QT}}$ power results from the observation that it is likely to be the consequence of the projection of cardiac axis movements due to respiration over a single lead given that it increased when assessed over $\mathrm{Z}$ lead compared to X and Y ones (Porta et al., 1998b) and it is present in subjects under cardiac pacing (Lombardi et al., 1996). The non-autonomic nature of the $\mathrm{HF}_{\mathrm{QT}}$ power was supported by the present study as well: indeed, it is invariable in both human and animal protocols.

The proposed strategy has the inherent limitation of disregarding the dependence of QTV on RRV due to the wellknown relation linking QT to the preceding RR (Bazett, 1929). However, the selection of spectral indexes computed in different frequency bands (i.e., $\mathrm{HF}_{\mathrm{RR}}$ and $\mathrm{LF}_{\mathrm{QT}}$ powers) should mitigate the effects of this dependence. Our result corroborates this observation given that in humans during R90 the HFRR power increased compared to REST, while the $\mathrm{LF}_{\mathrm{QT}}$ marker remained stable, and in rats only the $\mathrm{LF}_{\mathrm{QT}}$ power was greater in the AGG group compared to the non-AGG one while the $\mathrm{HF}_{\mathrm{RR}}$ power was unvaried. However, models of the dynamical dependence of QTV on RRV should be tested (Porta et al., 1998a, 2010) in future to understand whether some normalization procedure should be applied to better represent the genuine contribution of the sympathetic drive directed to the ventricles.

\section{RRV and QTV Can Be Fruitfully Exploited for Cardiac Autonomic Characterization in Rats}

To the best of our knowledge, this is the first study in which QTV analysis was carried out on rats with the aim at assessing cardiac autonomic control and QTV markers were discussed along with those derived from RRV analysis. This approach was successfully applied with the aim at differentiating WI and WT rats and divergent subpopulations within the WT strain. WI rats are highly domesticated, docile, and placid, while WT rats exhibit a more aggressive behavior during a social conflict (Buwalda et al., 2011) than WI rats. These differences in trait aggressiveness between the two strains are mirrored by a different state of the sympatho-vagal balance in unstressed conditions, with WT rats generally showing lower indexes of cardiac vagal modulation than WI counterparts (Carnevali and Sgoifo, 2014). Our results are in agreement with Carnevali and Sgoifo (2014) given that we found a lower $\mathrm{HF}_{\mathrm{RR}}$ power in WT rats than in WI rats. The expected increase of the LF $\mathrm{QT}_{\mathrm{T}}$ marker, suggesting a higher sympathetic control in WT rats than in WI animals, was not found even though a tendency toward an increase of the $\mathrm{LF}_{\mathrm{QT}}$ power was evident. Since in presence of an active sympathovagal balance it is expected that a significant increase of $\mathrm{HF}_{\mathrm{RR}}$ power is associated to a significant decrease of the $\mathrm{LF}_{\mathrm{QT}}$ one, the decrease of $\mathrm{HF}_{\mathrm{RR}}$ power in WT animals in association with an unvaried $\mathrm{LF}_{\mathrm{QT}}$ index might suggest a greater complexity of the interactions between vagal and sympathetic branches of the autonomic nervous system. Complex interactions between the two branches of the autonomic nervous system are known to lead to imbalanced situations in which a vagal withdrawal is not linked to a simultaneous and proportional sympathetic activation or vice versa (Porta et al., 2007) or situations featuring co-activation or co-inhibition of both the autonomic nervous system limbs (Kollai and Koizumi, 1979; Paton et al., 2005). These situations might lead to non-reciprocal trends in cardiac vagal and sympathetic controls (Kollai and Koizumi, 1979). The complexity of the sympatho-vagal interactions requires a more flexible tool that does not pretend to quantify cardiac autonomic control from a unique variability series like RRV-based analysis, but considers the joint observation of RRV and QTV as a mandatory standpoint for the reliable inference of autonomic nervous system state.

The relevance of the simultaneous assessment of RRV and QTV is even more evident when the WT rats were subdivided into non-AGG and AGG animals (de Boer et al., 2003). In previous studies, AGG rats were found to be characterized 
by lower RRV markers in unstressed conditions compared to non-AGG rats, thus suggesting that the aggressive behavior is associated with a lower vagal control (Carnevali et al., 2013; Carnevali and Sgoifo, 2014). Such a low cardiac vagal modulation was associated with a higher arrhythmia susceptibility and a greater vulnerability to cardiac morbidity in the AGG group (Carnevali et al., 2013). Differences in resting autonomic modulation between AGG and non-AGG rats were not evident in the current study using the RRV markers given that the $\mathrm{HF}_{\mathrm{RR}}$ power was similar, but they were unveiled by the QTV markers given that the $\mathrm{LF}_{\mathrm{QT}}$ power was greater in AGG than in nonAGG rats. Therefore, our results suggest that the AGG rats are characterized by a higher resting sympathetic modulation that is not accompanied by a concomitant reduction of vagal modulation. This finding might be another evidence of the complexity of the cardiac control in rats where a high sympathetic drive does not imply by necessity a vagal withdrawal and further corroborates the need of an approach to the study of the cardiac autonomic control integrating different signals and not necessarily based on the concept of sympatho-vagal balance.

\section{Time Domain RRV and QTV Parameters Versus Spectral RRV and QTV Markers}

Time domain markers were commonly shown to provide the representation of the effect of a physiological challenge or an experimental maneuver on the cardiovascular system. For example, in our human protocol, the trend of the $\mu_{R R}$ suggests that the orthostatic challenge was effective because the reduction of the venous return due to posture modification provokes a tachycardic response in the attempt to prevent the arterial pressure drop (Montano et al., 1994; Cooke et al., 1999; Porta et al., 2011; Marchi et al., 2016). For example, in the same protocol the evolution of $\mu_{\mathrm{QT}}$ suggests that the QT measures are reliable given that it is well-known that in humans $\mu_{\mathrm{QT}}$ is shorter when $\mu_{R R}$ is reduced (Bazett, 1929). However, the limits of time domain measures in providing a complete picture appear evidently as well. For example, $\sigma_{\mathrm{QT}}^{2}$ was less powerful than the $\mathrm{LF}_{\mathrm{QT}}$ power in describing the effect of the orthostatic challenge likely because non-autonomic effects resulting from cardiac axis movements synchronous with respiration (Porta et al., 1998b) are likely to influence more remarkably $\sigma_{\mathrm{QT}}^{2}$ than its portion in the LF band. For example, in non-AGG and AGG rats the $\mu_{\mathrm{RR}}$ and $\mu_{\mathrm{QT}}$ were similar, while the $\mathrm{LF}_{\mathrm{QT}}$ power increased in the AGG group, thus stressing the non-redundant nature of time and frequency domain markers.

\section{On the Use of Rats as an Animal Model of Human Cardiac Autonomic Control Explored via RRV and QTV Analyses}

Rats are considered animals exhibiting a sympathetic dominance given that their intrinsic heart rate (i.e., the cardiac frequency under complete pharmacological autonomic blockade) is lower than the resting heart rate (Opthof, 2000). However, this observation does not imply that vagal control is absent. Indeed, the full muscarinic receptor blockade induced via a high dose of atropine dramatically reduced RRV (Japundzic et al., 1990;
Cerutti et al., 1991; Silva et al., 2017), thus supporting the observation that changes of vagal activity contribute importantly to $\sigma_{\mathrm{RR}}^{2}$ and corroborating the use of these animals in translational studies on cardiac autonomic control. More importantly for the present study, rats respond differently to sympathetic stimulation: indeed, they show a QT prolongation, while in humans a QT shortening is observed (Conrath and Opthof, 2006; Speerschneider and Thomsen, 2013). The parallel changes of $\mu_{\mathrm{RR}}$ and $\mu_{\mathrm{QT}}$ reported in the present study in the human protocol and the opposite trends of $\mu_{\mathrm{RR}}$ and $\mu_{\mathrm{QT}}$ in WI and WT groups are in agreement with the diverse effect of an augmented sympathetic drive on $\mu_{\mathrm{RR}}$ and $\mu_{\mathrm{QT}}$ in humans and rats. In spite of this peculiarity, the RRV and QTV markers seem to maintain similar interpretation in both species. However, the lack of application of a stressor inducing a sympathetic activation in both WI and WT rats prevents us to deepen this issue.

\section{Limitations of the Study and Future Developments}

While our data support the association between QTV magnitude and sympathetic control, they are less informative about the shape of the relation between them. It is likely that the QTV could reflect mean sympathetic activity and its modifications about the mean when sympathetic drive is sufficiently high, while below a certain mean neural activity value QTV could be useless. We advocate pharmacological studies that could graduate the challenge in a finer manner and the contemporaneous direct recording of sympathetic activity to provide insight on the shape of this relation.

There is an open debate on the dependency of the magnitude of RRV and QTV on their means and on the need of some normalization (Sacha and Pluta, 2008; Sacha, 2013; Boyett et al., 2019; Malik et al., 2019). In the present study we tested the redundancy between QTV and $\mu_{\mathrm{QT}}$ by calculating the normalized QT variance (QTVN), namely the ratio of the square QT standard deviation to the square $\mu_{\mathrm{QT}}$ (Baumert et al., 2016). No difference was found either among experimental conditions in the human protocol or between groups in the animal protocol. This result might suggest a certain degree of dependency between QTV and $\mu_{\mathrm{QT}}$. However, the lack of significant differences is due to the enormous standard deviation of QTVN, sometimes close to two times the QTVN mean. This observation suggests some caution in using QTVN given that normalization procedure might behave differently at diverse values of $\mu_{\mathrm{QT}}$ and the need of more deeply exploring the relation between QTV and $\mu_{\mathrm{QT}}$.

Since in rats the T-wave morphology is different from that in humans, due to the different shapes of the ventricular action potentials (Fabritz et al., 2010; Boukens et al., 2014), future studies should be focused on the comparison of methods based on a threshold on the first derivative (Laguna et al., 1990; Nollo et al., 1992; Porta et al., 1998b), on the tangent method taking the interception between the straight line at the steepest point of the T-wave downslope and the isoelectric line (Lepeschkin and Surawicz, 1952; Yamada et al., 1993; Porta et al., 1998b) and on template matching approach (Berger et al., 1997; Baumert et al., 2012). 


\section{CONCLUSION}

In the present study, we computed frequency domain markers concurrently derived from RRV and QTV for a deeper characterization of the cardiac autonomic control. The power of RRV in the HF band and the power of QTV in the LF band were exploited to typify state- and trait-related modifications of the cardiac autonomic regulation in humans and rats. We found that the information derived from RRV and QTV spectral markers is not redundant given that trends of the HF power of RRV cannot be inferred from those of the LF power of QTV and vice versa. The complementary information was interpreted in relation to the inherent ability of RRV and QTV spectral markers to describe, respectively, cardiac vagal and sympathetic controls. Therefore, we conclude that the concomitant evaluation of RRV and QTV frequency domain markers can provide a more insightful view on cardiac autonomic function in both humans and rats than the sole exploitation of RRV indexes.

\section{DATA AVAILABILITY STATEMENT}

The datasets generated for this study are available on request to the corresponding author.

\section{REFERENCES}

Akaike, H. (1974). A new look at the statistical novel identification. IEEE Trans. Autom. Control 19, 716-723. doi: 10.1109/tac.1974.1100705

Akselrod, S., Eliash, S., Oz, O., and Cohen, S. (1987). Hemodynamic regulation in SHR: investigation by spectral analysis. Am. J. Physiol. 253, H176-H183.

Akselrod, S., Gordon, D., Ubel, F. A., Shannon, D. C., Berger, A. C., and Cohen, R. J. (1981). Power spectrum analysis of heart rate fluctuation: a quantitative probe of beat-to-beat cardiovascular control. Science 213, 220-222. doi: 10. $1126 /$ science.6166045

Atiga, W. L., Calkins, H., Lawrence, J. H., Tomaselli, G. F., Smith, J. M., and Berger, R. D. (1998). Beat-to-beat repolarization lability identifies patients at risk for sudden cardiac death. J. Cardiovasc. Electrophysiol. 9:908.

Bar, K. J., Koschke, M., Boettger, M. K., Berger, S., Kabisch, A., Sauer, H., et al. (2007). Acute psychosis leads to increased QT variability in patients suffering from schizophrenia. Schizophr. Res. 95, 115-123. doi: 10.1016/j.schres.2007. 05.034

Bari, V., Valencia, J. F., Vallverdú, M., Girardengo, G., Marchi, A., Bassani, T., et al. (2014). Multiscale complexity analysis of the cardiac control identifies asymptomatic and symptomatic patients in long QT syndrome type 1. PLoS One 9:e93808. doi: 10.1371/journal.pone.0093808

Baselli, G., Porta, A., Rimoldi, O., Pagani, M., and Cerutti, S. (1997). Spectral decomposition in multichannel recordings based on multi-variate parametric identification. IEEE Trans. Biomed. Eng. 44, 1092-1101. doi: 10.1109/10. 641336

Baumert, M., Porta, A., Vos, M. A., Malik, M., Couderc, J. P., Laguna, P., et al. (2016). QT interval variability in body surface ECG: measurement, physiological basis, and clinical value: position statement and consensus guidance endorsed by the european heart rhythm association jointly with the esc working group on cardiac cellular electrophysiology. Europace 186, 925-944. doi: 10.1093/europace/euv405

Baumert, M., Lambert, G. W., Dawood, T., Lambert, E. A., Esler, M. D., McGrane, M., et al. (2008). QT interval variability and cardiac norepinephrine spillover in patients with depression and panic disorder. Am. J. Physiol. 295, H962-H968. doi: 10.1152/ajpheart.00301.2008

\section{ETHICS STATEMENT}

The studies involving human participants were reviewed and approved by the Human Research and Ethical Review Board of the L. Sacco Hospital, Milan, Italy. The patients/participants provided their written informed consent to participate in this study. The animal study was reviewed and approved by the Veterinarian Animal Care and Use Committee of the University of Parma, Parma, Italy.

\section{AUTHOR CONTRIBUTIONS}

AP conceived and designed the study. AS, LC, AMT, and AC performed the experiments. $\mathrm{BM}$ analyzed the data. $\mathrm{BM}$ and $\mathrm{AP}$ drafted the manuscript and prepared the figures. $\mathrm{BM}, \mathrm{VB}, \mathrm{AS}$, LC, BC, EV, AMT, AC, LDV, and AP interpreted the results, edited and revised the manuscript, and approved the final version of the manuscript.

\section{FUNDING}

This work was partially supported by the Ricerca Corrente from the Ministry of Health, Italy.

Baumert, M., Schlaich, M. P., Nalivaiko, E., Lambert, E., Sari, C. I., Kaye, D. M., et al. (2011). Relation between QT interval variability and cardiac sympathetic activity in hypertension. Am. J. Physiol. 300, H1412-H1417.

Baumert, M., Starc, V., and Porta, A. (2012). Conventional QT variability measurement vs. template matching techniques: comparison of performance using simulated and real ECG. PLoS One 7:e41920. doi: 10.1371/journal.pone. 0041920

Bazett, H. C. (1929). An analysis of the time-relations of electrocardiograms. Heart 7, 353-370.

Berger, R. D. (2009). QT Interval variability: is it a measure of autonomic activity? J. Am. Coll. Cardiol. 54, 821-852.

Berger, R. D., Kasper, E. K., Baughman, K. L., Marban, E., Calkins, H., and Tomaselli, G. F. (1997). Beat-to-beat QT interval variability: novel evidence for repolarization lability in ischemic and nonischemic dilated cardiomyopathy. Circulation 96, 1557-1565. doi: 10.1161/01.cir.96.5.1557

Billman, G. E. (2013). The LF/HF ratio does not accurately measure cardiac sympatho-vagal balance. Front. Physiol. 4:26. doi: 10.3389/fphys.2013.00026

Boukens, B. J., Rivaud, M. R., Rentschler, S., and Coronel, R. (2014). Misinterpretation of the mouse ECG: 'musing the waves of Mus musculus'. J. Physiol. 592, 4613-4626. doi: 10.1113/jphysiol.2014.279380

Boyett, M., Wang, Y., and D'Souza, A. (2019). CrossTalk opposing view: heart rate variability as a measure of cardiac autonomic responsiveness is fundamentally flawed. J. Physiol. 597, 2599-2601. doi: 10.1113/jp277501

Malik, M., Hnatkova, K., Huikuri, H. V., Lombardi, F., Schmidt, G., and Zabel, M. (2019). CrossTalk proposal: heart rate variability is a valid measure of cardiac autonomic responsiveness. J. Physiol. 597, 2595-2598. doi: 10.1113/jp2 77500

Brenner, I. K., Thomas, S., and Shephard, R. J. (1997). Spectral analysis of heart rate variability during heat exposure and repeated exercise. Eur. J. Appl. Physiol. Occup. Physiol. 76, 145-156. doi: 10.1007/s004210050227

Buwalda, B., Geerdink, M., Vidal, J., and Koolhaas, J. M. (2011). Social behavior and social stress in adolescence: a focus on animal models. Neurosci. Biobehav. Rev. 35, 1713-1721. doi: 10.1016/j.neubiorev.2010.10.004

Carnevali, L., and Sgoifo, A. (2014). Vagal modulation of resting heart rate in rats: the role of stress, psychosocial factors, and physical exercise. Front. Physiol. 5:118. doi: $10.3389 /$ fphys. 2014.00118 
Carnevali, L., Trombini, M., Porta, A., Montano, N., de Boer, S. F., and Sgoifo, A. (2013). Vagal withdrawal and susceptibility to cardiac arrhythmias in rats with high trait aggressiveness. PLoS One 87:e68316. doi: 10.1371/journal.pone. 0068316

Cerutti, C., Gustin, M. P., Paultre, C. Z., Lo, M., Julien, C., Vincent, M., et al. (1991). Autonomic nervous system and cardiovascular variability in rats: a spectral analysis approach. Am. J. Physiol. 261, H1292-H1299.

Conrath, C. E., and Opthof, T. (2006). Ventricular repolarization: an overview of (patho)physiology, sympathetic effects and genetic aspects. Prog. Biophys. Mol. Biol. 92, 269-307. doi: 10.1016/j.pbiomolbio.2005.05.009

Cooke, W. H., Hoag, J. B., Crossman, A. A., Kuusela, T. A., Tahvanainen, K. U. O., and Eckberg, D. L. (1999). Human responses to upright tilt: a window on central autonomic integration. J. Physiol. 517, 617-628. doi: 10.1111/j.1469-7793.1999. 0617t.x

de Boer, S. F., van der Vegt, B. J., and Koolhaas, J. M. (2003). Individual variation in aggression of feral rodent strains: a standard for the genetics of aggression and violence? Behav. Genet. 33, 485-501.

Eckberg, D. L. (1997). Sympathovagal balance: a critical appraisal. Circulation 96, 3224-3232. doi: 10.1161/01.cir.96.9.3224

El-Hamad, F., Lambert, E., Abbott, D., and Baumert, M. (2015). Relation between QT interval variability and muscle sympathetic nerve activity in normal subjects. Am. J. Physiol. 309, H1218-H1224. doi: 10.1152/ajpheart.00230.2015

Fabritz, L., Damke, D., Emmerich, M., Kaufmann, S. G., Theis, K., Blana, A., et al. (2010). Autonomic modulation and antiarrhythmic therapy in a model of long QT syndrome type 3. Cardiovasc. Res. 87, 60-72. doi: 10.1093/cvr/cvq029

Hirsch, J. A., and Bishop, B. (1981). Respiratory sinus arrhythmia in humans: how breathing pattern modulates heart rate. Am. J. Physiol. 241, H620-H629.

Jaenisch, R. B., Hentschke, V. S., Quagliotto, E., Cavinato, P. R., Schmeing, L., Xavier, L. L., et al. (2011). Respiratory muscle training improves hemodynamics, autonomic function, baroreceptor sensitivity, and respiratory mechanics in rats with heart failure. J. Appl. Physiol. 111, 1664-1670. doi: 10.1152/japplphysiol. 01245.2010

Japundzic, N., Grichois, M. L., Zitoun, P., Laude, D., and Elghozi, J. L. (1990). Spectral analysis of blood pressure and heart rate in conscious rats: effects of autonomic blockers. J. Auton. Nerv. Syst. 30, 91-100. doi: 10.1016/01651838(90)90132-3

Kay, S. M., and Marple, S. L. (1981). Spectrum analysis: a modern perspective. Proc. IEEE 69, 1380-1418.

Kollai, M., and Koizumi, K. (1979). Reciprocal and non-reciprocal action of the vagal and sympathetic nerves innervating the heart. J. Auton. Nerv. Syst. 1, 33-52. doi: 10.1016/0165-1838(79)90004-3

Laguna, P., Thakor, N. V., Caminal, P., Jane, R., Yoon, H. R., Bayes de Luna, A., et al. (1990). New algorithm for QT interval analysis in 24-hour Holter ECG: performance and applications. Med. Biol. Eng. Comput. 281, 67-73. doi: $10.1007 /$ bf02441680

Lepeschkin, E., and Surawicz, B. (1952). The measurement of the Q-T interval of the electrocardiogram. Circulation 6, 378-388. doi: 10.1161/01.cir.6.3.378

Lombardi, F., Sandrone, G., Porta, A., Torzillo, D., Terranova, G., Baselli, G., et al. (1996). Spectral analysis of short term R-Tapex interval variability during sinus rhythm and fixed atrial rate. Eur. Heart J. 17, 769-778. doi: 10.1093/ oxfordjournals.eurheartj.a014945

Magagnin, V., Bassani, T., Bari, V., Turiel, M., Maestri, R., Pinna, G. D., et al. (2011). Non-stationarities significantly distort short-term spectral, symbolic and entropy heart rate variability indexes. Physiol. Meas. 32, 1775-1786. doi: $10.1088 / 0967-3334 / 32 / 11 /$ s05

Malik, M. (2009). Beat-to-beat QT variability and cardiac autonomic regulation. Am. J. Physiol. 295, H923-H925.

Marchi, A., Bari, V., De Maria, B., Esler, M., Lambert, E., Baumert, M., et al. (2016). Calibrated variability of muscle sympathetic nerve activity during graded headup tilt in humans and its link with noradrenaline data and cardiovascular rhythms. Am J. Physiol. 310, R1134-R1143. doi: 10.1152/ajpregu.00541.2015

Montano, N., Gnecchi-Ruscone, T., Porta, A., Lombardi, F., Pagani, M., and Malliani, A. (1994). Power spectrum analysis of heart rate variability to assess changes in sympatho-vagal balance during graded orthostatic tilt. Circulation 90, 1826-1831. doi: 10.1161/01.cir.90.4.1826

Nollo, G., Speranza, G., Grasso, R., Bonamini, R., Mangiardi, L., and Antolini, R. (1992). Spontaneous beat-to-beat variability of the ventricular repolarisation duration. J. Electrocardiol. 25, 9-17. doi: 10.1016/0022-0736(92)90124-i
Opthof, T. (2000). The normal range and determinants of the intrinsic heart rate in man. Cardiovasc. Res. 45, 177-184. doi: 10.1016/s0008-6363(99)00322-3

Pagani, M., Lombardi, F., Guzzetti, S., Rimoldi, O., Furlan, R., Pizzinelli, P., et al. (1986). Power spectral analysis of heart rate and arterial pressure variabilities as a marker of sympatho-vagal interaction in man and conscious dog. Circ. Res. 59, 178-193. doi: 10.1161/01.res.59.2.178

Pagani, M., Montano, N., Porta, A., Malliani, A., Abboud, F. M., Birkett, C., et al. (1997). Relationship between spectral components of cardiovascular variabilities and direct measures of muscle sympathetic nerve activity in humans. Circulation 95, 1441-1448. doi: 10.1161/01.cir.95. 6.1441

Paton, J. F., Boscan, P., Pickering, A. E., and Nalivaiko, E. (2005). The yin and yang of cardiac autonomic control: vago-sympathetic interactions revisited. Brain Res. Rev. 49, 555-565. doi: 10.1016/j.brainresrev.2005.02.005

Piccirillo, G., Cacciafesta, M., Lionetti, M., Nocco, M., Di Giuseppe, V., Moisé, A., et al. (2001). Influence of age, the autonomic nervous system and anxiety on QT-interval variability. Clin. Sci. 101, 429-438. doi: 10.1042/cs10 10429

Piccirillo, G., Magnanti, M., Matera, S., Di Carlo, S., De Laurentis, T., Torrini, A., et al. (2006). Age and QT variability index during free breathing, controlled breathing and tilt in patients with chronic heart failure and healthy control subjects. Transl. Res. 142, 72-78. doi: 10.1016/j.trsl.2006.02.001

Pomeranz, B., Macaulay, R. J. B., Caudill, M. A., Kutz, I., Adam, D., Gordon, D., et al. (1985). Assessment of autonomic function in humans by heart-rate spectral-analysis. Am. J. Physiol. 248, H151-H153.

Porta, A., Bari, V., Badilini, F., Tobaldini, E., Gnecchi-Ruscone, T., and Montano, N. (2011). Frequency domain assessment of the coupling strength between ventricular repolarization duration and heart period during graded head-up tilt. J. Electrocardiol. 44, 662-668. doi: 10.1016/j.jelectrocard.2011.08.002

Porta, A., Bari, V., De Maria, B., Cairo, B., Vaini, E., Malacarne, M., et al. (2018). Peripheral resistance baroreflex during incremental bicycle ergometer exercise: characterization and correlation with cardiac baroreflex. Front. Physiol. 9:688. doi: 10.3389/fphys.2018.00688

Porta, A., Baselli, G., Caiani, E., Malliani, A., Lombardi, F., and Cerutti, S. (1998a). Quantifying electrocardiogram RT-RR variability interactions. Med. Biol. Eng. Comput. 36, 27-34. doi: 10.1007/bf02522854

Porta, A., Baselli, G., Lombardi, F., Cerutti, S., Antolini, R., Del Greco, M., et al. (1998b). Performance assessment of standard algorithms for dynamic R-T interval measurement: comparison between R-Tapex and R-Tend approach. Med. Biol. Eng. Comput. 36, 35-42. doi: 10.1007/bf02522855

Porta, A., Girardengo, G., Bari, V., George, A. L. Jr., Brink, P. A., Goosen, A., et al. (2015). Autonomic control of heart rate and QT interval variability influences arrhythmic risk in long QT syndrome type I. J. Am. Coll. Cardiol. 65, 367-374. doi: 10.1016/j.jacc.2014.11.015

Porta, A., Tobaldini, E., Gnecchi-Ruscone, T., and Montano, N. (2010). RT variability unrelated to heart period and respiration progressively increases during graded head-up tilt. Am. J. Physiol. 298, H1406-H1414. doi: 10.1152/ ajpheart.01206.2009

Porta, A., Tobaldini, E., Guzzetti, S., Furlan, R., Montano, N., and GnecchiRuscone, T. (2007). Assessment of cardiac autonomic modulation during graded head-up tilt by symbolic analysis of heart rate variability. Am. J. Physiol. 293, H702-H708.

Reyes del Paso, G. A., Langewitz, W., Mulder, L. J., van Roon, A., and Duschek, S. (2013). The utility of low frequency heart rate variability as an index of sympathetic cardiac tone: a review with emphasis on a reanalysis of previous studies. Psychophysiology 50, 477-487. doi: 10.1111/psyp.12027

Rubini, R., Porta, A., Baselli, G., Cerutti, S., and Paro, M. (1993). Power spectrum analysis of cardiovascular variability monitored by telemetry in conscious unrestrained rats. J. Auton. Nerv. Syst. 45, 181-190. doi: 10.1016/0165-1838(93) 90050-5

Sacha, J. (2013). Why should one normalize heart rate variability with respect to average heart rate. Front. Physiol. 4:306. doi: 10.3389/fphys.2013. 00306

Sacha, J., and Pluta, W. (2008). Alterations of an average heart rate change heart rate variability due to mathematical reasons. Int. J. Cardiol. 128, 444-447. doi: 10.1016/j.ijcard.2007.06.047

Sgoifo, A., De Boer, S. F., Buwalda, B., Korte-Bouws, G., Tuma, J., Bohus, B., et al. (1998). Vulnerability to arrhythmias during social stress in rats with different 
sympathovagal balance. Am. J. Physiol. 275, H460-H466. doi: 10.1152/ajpheart. 1998.275.2.H460

Sgoifo, A., Koolhaas, J., De Boer, S., Musso, E., Stilli, D., Buwalda, B., et al. (1999). Social stress, autonomic neural activation, and cardiac activity in rats. Neurosci. Biobehav. Rev. 237, 915-923. doi: 10.1016/s0149-7634(99)00025-1

Shin, K., Minamitani, H., Onishi, S., Yamazaki, H., and Lee, M. (1995a). The power spectral analysis of heart rate variability in athletes during dynamic exercise Part I. Clin. Cardiol. 18, 583-586. doi: 10.1002/clc.4960181011

Shin, K., Minamitani, H., Onishi, S., Yamazaki, H., and Lee, M. (1995b). The power spectral analysis of heart rate variability in athletes during dynamic exercise Part II. Clin. Cardiol. 18, 664-668. doi: 10.1002/clc.4960181114

Silva, L. E. V., Lataro, R. M., Castania, J. A., da Silva, C. A. A., Valencia, J. F., Murta, L. O. Jr., et al. (2016). Multiscale entropy analysis of heart rate variability in heart failure, hypertensive and sinoaortic-denervated rats: classical and refined approaches. Am. J. Physiol. 310, R150-R156. doi: 10.1152/ajpregu.00076.2016

Silva, L. E. V., Rezende Geraldini, V., Potratz de Oliveira, B., Aguiar Silva, C. A., Porta, A., and Fazan, R. (2017). Comparison between spectral analysis and symbolic dynamics for heart rate variability analysis in the rat. Sci. Rep. 7:8428. doi: 10.1038/s41598-017-08888-w

Speerschneider, T., and Thomsen, M. B. (2013). Physiology and analysis of the electrocardiographic T wave in mice. Acta Physiol. 209, 262-271. doi: 10.1111/ apha. 12172

Speranza, G., Nollo, G., Ravelli, F., and Antolini, R. (1993). Beat-to-beat measurement and analysis of the R-T interval in $24 \mathrm{~h}$ ECG Holter recordings. Med. Biol. Eng. Comput. 315, 487-494. doi: 10.1007/bf02441984

Stauss, H. M., Persson, P. B., Johnson, A. K., and Kregel, K. C. (1997). Frequency response characteristics of autonomic nervous system function in conscious rats. Am. J. Physiol. 273, H786-H795.
Task Force of the European Society of Cardiology and the North American Society of Pacing and Electrophysiology, (1996). Heart rate variability. Standards of measurement, physiological interpretation, and clinical use. Eur. Heart. J. 17, 354-381. doi: 10.1093/oxfordjournals.eurheartj.a01 4868

Yamada, A., Hayano, J., Horie, K., Ieda, K., Mukai, S., Yamada, M., et al. (1993). Regulation of QT interval during postural transitory changes in heart rate in normal subjects. Am. J. Cardiol. 71, 996-998. doi: 10.1016/0002-9149(93) 90922-y

Yeragani, V. K., Pohl, R., Jampala, V. C., Balon, R., Kay, J., and Igel, G. (2000a). Effect of posture and isoproterenol on beat-to-beat heart rate and QT variability. Neuropsychobiology 41, 113-123. doi: 10.1159/000026642

Yeragani, V. K., Pohl, R., Jampala, V. C., Balon, R., Ramesh, C., and Srinivasan, K. (2000b). Increased QT variability in patients with panic disorder and depression. Psychiatry Res. 93, 225-235. doi: 10.1016/s0165-1781(00) 00119-0

Conflict of Interest: The authors declare that the research was conducted in the absence of any commercial or financial relationships that could be construed as a potential conflict of interest.

Copyright ( 2019 De Maria, Bari, Sgoifo, Carnevali, Cairo, Vaini, Catai, de Medeiros Takahashi, Dalla Vecchia and Porta. This is an open-access article distributed under the terms of the Creative Commons Attribution License (CC BY). The use, distribution or reproduction in other forums is permitted, provided the original author(s) and the copyright owner(s) are credited and that the original publication in this journal is cited, in accordance with accepted academic practice. No use, distribution or reproduction is permitted which does not comply with these terms. 